\title{
Ribbon Element on Co-Frobenius Quasitriangular Hopf Algebras
}

\author{
Guohua Liu \\ Department of Mathematics, Southeast University, Nanjing, China \\ E-mail: liuguohua2000cn@yahoo.com.cn \\ Received June 5, 2010; revised July 23, 2010; accepted July 29, 2010
}

\begin{abstract}
Let $(H, R)$ be a co-Frobenius quasitriangular Hopf algebra with antipode $S$. Denote the set of group-like elements in $H$ by $G(H)$. In this paper, we find a necessary and sufficient condition for $(H, R)$ to have a ribbon element. The condition gives a connection with the order of $G(H)$ and the order of $S^{2}$.
\end{abstract}

Keywords: Co-Frobenius Hopf Algebra, Ribbon Element

\section{Introduction}

A Hopf algebra $H$ is called co-Frobenius if $H$ is either left or right co-Frobenius as a coalgebra, i.e., if there exists a left or right $H^{*}$ monomorphism from $H$ to $H^{*}$. It turns out that $H$ is co-Frobenius if and only if $H$ has nonzero integrals $[1,2]$; in particular every finite dimensional Hopf algebra is co-Frobenius. Among the properties of finite dimensional Hopf algebras that hold for all co-Frobenius Hopf algebras are the bijectivity of the antipode, a bijective correspondence between the group-like elements of the Hopf algebra and the one dimensional ideals of the dual algebra, the existence of a distinguished group-like element, and a reasonable theory of Galois extensions.

The class of infinite dimensional co-Frobenius Hopf algebras includes cosemisimple Hopf algebras, such as the group algebra of an infinite group. Tensoring such a Hopf algebra $H$ with a finite dimensional Hopf algebra $K$, yields an infinite dimensional Hopf algebra with non-zero integral obtained by tensoring the integrals of $H$ and $K$. As well, a recent example of Van Daele [3] gives an infinite dimensional co-Frobenius Hopf algebra without normal Hopf subalgebra.

The topological motivation for this paper is supported by the fact that ribbon Hopf algebras (Hopf algebra with a distinguished ribbon element) can be used to construct invariants of framed links embedded in three dimensional space [4]. And the same structure can be used to produce invariant of three dimensional manifolds. These three dimensional manifolds are represented by surgery on framed links, and their invariants are special cases of invariants for the links. In the case of quantum group $\mathrm{SLq}(2)$, these invariants have been intensively investigated by Reshetukhin and Turaev [5], Kirby [6], and others.

In this paper, we give a necessary and sufficient condition for the co-Frobenius quasitriangular Hopf algebra to have a ribbon element. Based on the ideals and results of Beattie, Bulacu and others [7-9], we generalize the results of Kauffman and Radford [10] to co-Frobenius quasitriangular Hopf algebras. We find the group-like elements $\alpha$ and $\mathrm{g}$ which play a special role in the theory of ribbon Hopf algebras. Our main result is Theorem 5, which states that a co-Frobenius quasitriangular Hopf algebra $(H, R)(G(H)$ has odd order) has a ribbon element if and only if, $S^{2}$ has odd order.

Throughout this paper, $H$ will denote a co-Frobenius Hopf algebra over a field $k$. All maps are assumed to be $k$-linear. We use the Sweedler-type notation for the comultiplication maps $\Delta(h)=h_{1} \otimes h_{2}$ for all $h \in H$. As usual, the $H^{*}$-bimodule structure on $H$ and the $H$-bimodule structure on $H^{*}$ are given by

$$
\begin{gathered}
1^{*} \rightarrow h \leftarrow m^{*}=m^{*}\left(h_{1}\right) h_{2} l^{*}\left(h_{3}\right) \\
\left(h \rightarrow m^{*} \leftarrow l\right)(m)=m^{*}(l m h)
\end{gathered}
$$

for all $h, l, m \in H$ and $l^{*}, m^{*} \in H^{*}$. The antipode of $\mathrm{H}$ is denoted by $S$ with composition inverse $S^{-1}$. The set of group-like elements in $\mathrm{H}$ is denoted by $\mathrm{G}(\mathrm{H})$ and the group-likes of $H^{0}$, namely the set of algebra maps from $H$ to $k$, by $G\left(H^{0}\right)$.

Let $H$ be a co-Frobenius Hopf algebra over a field $k$. Recall that a Hopf algebra $H$ is co-Frobenius if $H^{* r a t}$, 
the unique maximal rational submodule of $H^{*}$, is nonzero, or, equivalently, if the space of left or right integrals for $H$, denoted by $\int_{l}^{H^{*}}$ and $\int_{r}^{H^{*}}$ respectively, is nonzero. It was shown in [9] that $\stackrel{r}{H}$ contains a distinguished group-like element $g$, which is also called the modular element of $H$, such that for all $\lambda \in \int_{l}^{H^{*}}$ and $h \in H$ :

$$
\lambda\left(\mathrm{h}_{1}\right) h_{2}=\lambda(h) g^{-1} \text { and } \lambda \cdot S^{2}=g^{-1} \rightarrow \lambda \leftarrow g
$$

For $\Gamma$ either a nonzero left or right integral for $H$ in $H^{* \text { rat }}$, there are bijective maps from $H$ to $H^{* r a t}$ given by

$$
h \rightarrow(h \rightarrow \Gamma) \text { and } h \rightarrow(\Gamma \leftarrow h) .
$$

Let $\chi$ denote the generalized Frobenius automorphism of $H$ defined in [7], that is, for $\lambda \in \int_{l}^{H^{*}}, \chi$ is the algebra automorphism of $H$ defined by

$$
h \rightarrow \lambda=\lambda \leftarrow \chi(h) \text {, for all } h \in H .
$$

Then the algebra map $\alpha=\varepsilon \cdot \chi \in H^{*}$ is called the modular element for $H$ in $H^{*}$, and

$$
\chi(h)=\alpha\left(\mathrm{h}_{2}\right) S^{-2}\left(\mathrm{~h}_{1}\right), \text { for all } h \in H .
$$

Recall that, for a Hopf algebra $H$ and $R=R^{1} \otimes R^{2}=r^{1} \otimes r^{2} \in H \otimes H$, then $(H, R)$ is called quasitriangular if for all $h \in H$,

$$
\begin{aligned}
& (Q T 1) \Delta\left(R^{1}\right) \otimes R^{2}=R^{1} \otimes r^{1} \otimes R^{2} r^{2} \\
& (Q T 2) R^{1} \otimes \Delta\left(R^{2}\right)=R^{1} r^{1} \otimes r^{2} \otimes R^{2} \\
& (Q T 3)\left(\Delta^{c o p}(h)\right) R=R(\Delta(h)), \text { for all } h \in H ; \\
& (Q T 4) \varepsilon\left(R^{2}\right) R^{1}=1, \varepsilon\left(R^{1}\right) R^{2}=1 .
\end{aligned}
$$

Set

$$
u=S\left(R^{2}\right) R^{1}, c=u S(u) .
$$

By the result of Drinfeld [11] or Radford [12], $S^{2}$ is an inner automorphism induced by $u$ and $S(u)^{-1}$, i.e.

$$
S^{2}(a)=u a u^{-1}, \text { and } S^{2}(a)=S(u)^{-1} a S(u),
$$

for all $a \in H$.

$c$ is called the Casimir element of $(H, R)$, and $S^{2}(a)=u a u^{-1}$ implies that $c$ is in the center of $H$.

If $(H, R)$ is quasitriangular, Beattie and Bulauc [13] introduced two group homomorphisms from $G\left(H^{0}\right)$ to $G(H)$ given by

$$
\begin{aligned}
\eta \rightarrow a_{\eta} & :=\eta\left(R^{1}\right) R^{2}, \eta \rightarrow b_{\eta}:=\eta\left(S^{-1}\left(R^{2}\right)\right) R^{1} \\
& =\eta^{-1}\left(R^{2}\right) R^{1}
\end{aligned}
$$

They showed that $a_{\alpha}, b_{\alpha} \in G(H)$ and $a_{\alpha} b_{\alpha-1} \in$ $G(H) \cap Z(H)$. Now set

$$
g_{\alpha}=b_{\alpha-1}=\left(b_{\alpha}\right)^{-1}, h=b_{\alpha-1} g^{-1} .
$$

By [13], we have $u S(u)^{-1}=S(u)^{-1} u=g g_{\alpha}$.

By [12] and [13], we have $(g, \alpha$ denote the modular elements),

$$
c=u^{2} h \text {. }
$$

Since $c$ is central, $S^{2}(a)=u a u^{-1}, c=u^{2} h$, implies that

$$
\begin{aligned}
S^{4}(a) & =u S^{2}(a) u^{-1}=u^{2} a\left(u^{-1}\right)^{2} \\
& =u^{2} a c^{-1}\left(u^{-1}\right)^{2}=h^{-1} a h
\end{aligned}
$$

We say that $v \in H$ is a quasi-ribbon element of $(H, R)$ if the following conditions are satisfied:

$$
\begin{aligned}
& (R .1) v^{2}=c ; \\
& (R .2) S(v)=v ; \\
& (R .3) \varepsilon(v)=1 ; \\
& (R .4) \Delta(v)=\left(R_{21} R_{12}\right)^{-1}(v \otimes v),
\end{aligned}
$$

Drinfeld observed that $u$ satisfies the last condition. A quasi-ribbon element in the center of $H$ is called a ribbon element, and in this case $(H, R, v)$ is called a ribbon Hopf algebra [14]. The reader is referred to [14] for a detailed discussion of ribbon Hopf algebras and their relationship to links and three-manifolds.

\section{Ribbon Hopf Algebra}

Let $(H, R)$ be a finite dimensional quasitriangular Hopf algebra. In [10], the authors found a necessary and sufficient condition for the existence of ribbon elements on $(H, R)$. The purpose of this section is to generalize their result to co-Frobenius quasitriangular Hopf algebras. We find that most of the results in [10] also hold for coFrobenius quasitriangular Hopf algebras.

Lemma 1. Suppose that $(H, R)$ is a co-Frobenius quasitriangular Hopf algebra over a field $k, H$ contains a distinguished group-like element $g$ and that $\mathrm{v} \in H$ is a quasi-ribbon element of $\mathrm{H}$. Let

$$
g_{\alpha}=b_{\alpha-1}=\left(b_{\alpha}\right)^{-1}, h=b_{\alpha-1} g^{-1}, u=S\left(R^{2}\right) R^{1}
$$

and set $l=u^{-1} v$. Then:

1) $l^{2}=h$;

2) $l \in G(H)$.

Proof. 1) By (R.2) $(S(v)=v)$, we have $S^{2}(v)=v$. Thus $u$ and $v$ commute by $S^{2}(\alpha)=u a u^{-1}$ for all $a \in H$. By $(R .1) v^{2}=c$ and $c=u^{2} h$ we have $v^{2}=u^{2} h$. Thus $l^{2}=h$. 


$$
\begin{aligned}
\Delta(l) & =\Delta\left(u^{-1} v\right)=\Delta\left(u^{-1}\right) \Delta(v) \\
& =\Delta(u)^{-1} \Delta(v) \\
& =\left(\left(R_{21} R_{12}\right)^{-1} u \otimes u\right)^{-1}\left(R_{21} R_{12}\right)^{-1}(v \otimes v) \\
& =u^{-1} v \otimes u^{-1} v=l \otimes l
\end{aligned}
$$

Theorem 2. Suppose that $(H, R)$ is a co-Frobenius quasitriangular Hopf algebra over a field $k$, and let $u$ and $v$ be as above. Then:

1) $l \rightarrow u l$ defines a one-one correspondence between $\left\{l \in G(H) \mid l^{2}=h\right\} \leftrightarrow\{$ quasi-ribbon elements of $(H, R)\}$;

2) Suppose that $l \in G(H)$ and $l^{2}=h$, Then $v=u l$ is a ribbon element of $(H, R)$ if and only if $S^{2}(a)=l^{-1}$ al for all $a \in H$.

Proof. 1) Recall that $u$ commutes with the group-like elements of $H$. Thus $v=u l=l u$. Using $\left(u^{2} h=c\right)$ we see that $v^{2}=u^{2} l^{2}=u^{2} h=c$, so (R.1) holds for $v$. Now,

$$
S(v)=S(u l)=S(l) S(u)=l^{-1} S(u),
$$

by $l^{2}=h$ we have $l^{-1}=l h^{-1}$ and $h u=S(u)$, which follows from $u^{2} h=c=u S(u)$, Therefore

$$
S(v)=l^{-1} S(u)=l^{-1} h u=l h^{-1} h u=l u=u l=v .
$$

Thus, (R.2) holds for $v$. Note that (R.3) is immediate since $\varepsilon(u)=1=\varepsilon(l)$. Also

$$
\begin{aligned}
\Delta(v) & =\Delta(u l)=\Delta(u) \Delta(l)=\left(R_{21} R_{12}\right)^{-1}(u \otimes u)(l \otimes l) \\
& =\left(R_{21} R_{12}\right)^{-1} v \otimes v
\end{aligned}
$$

and (R.4) holds for $v$. The proof of (1) is finished by Lemma 1 .

2) If $v=u l, S^{2}(a)=u a u^{-1}=\left(v l^{-1}\right) a\left(v l^{-1}\right)^{-1}=l^{-1} a l$. On the other hand, $S^{2}(a)=l^{-1} a l=u a u^{-1}$ implies $v a=$ $a v$ for all $a \in H$.

Corollary 3. Suppose that $(H, R)$ is a co-Frobenius quasitriangular Hopf algebra. Let $g$ and $a$ be the distinguished group-like elements of $H$ and $H^{*}$, respectively, and let $h$ be as above. Then:

1) If $h$ has odd order, or if $g$ and a have odd order, Then $(H, R)$ has a quasi-ribbon element;

2) If $G(H)$ has odd order, Then $(H, R)$ has a unique quasi-ribbon element.

Proof. 1) By $b_{a-1}$ commuting with all $a \in G(H)$, and $\eta \rightarrow b_{\eta}$ is a group homomorphism from $G\left(H^{0}\right)$ to $G(H)$. We have that $h$ has a square root in $G(H)$, which must be unique if $G(H)$ has odd order. Therefore the corollary follows by part 1 of the above Theorem.

Proposition 4. Suppose that $(H, R)$ is a co-Frobenius quasitriangular Hopf algebra. Let $g$ and $a$ be the distinguished group-like elements of $H$ and $H^{*}$, respectively, and let $h$ be as above. Then if either

1) If $h$ and $S^{2}$,or;
2) If $g, a$ and $S^{2}$ have odd order. Then $(H, R)$ has a ribbon element.

Proof. First, condition (2) implies condition (1). Suppose that $h$ and $S^{2}$ have odd order. Let $l$ be the unique square root of $h$ having odd order. Define map $\tau(a): H \rightarrow H$ by $\tau(a)(b)=a b a^{-1}$.

Then $\tau\left(l^{-1}\right)^{2}=\tau\left(h^{-1}\right)$ and $\tau\left(l^{-1}\right)$ have odd order. Recall that $S^{4}(a)=h^{-1} a h=\tau\left(h^{-1}\right)$. Since $l \in G(H)$, $S^{2}$ and $\tau\left(l^{-1}\right)$ are two elements of odd order whose squares are equal. Consequently, $S^{2}=\tau\left(l^{-1}\right)$, and the proposition follows by part (2) of Theorem 2 .

Theorem 5. Suppose that $(H, R)$ is a co-Frobenius quasitriangular Hopf algebra with antipode $S$ over a field $k$ and assume that $G(H)$ has odd order, Then $(H, R)$ has a ribbon element (which is necessarily unique) if and only if $S^{2}$ has odd order.

Proof. If $(H, R)$ has a ribbon element, then there exists an $x \in G(H)$ such that $S^{2}(a)=x a x^{-1}$ for all $a \in H$ by Theorem 2 . Since $x$ has odd order it follows that $\mathrm{S}^{2}$ does also.

Conversely, suppose that $S^{2}$ has odd order. Since $h$ has odd order, it follows that $(H, R)$ has a ribbon element by Proposition 4 . This completes our proof.

When $H$ is unimodular, We note that $h=g^{-1}$ since $a=\varepsilon$ in this case, by Theorem 2, the existence of ribbon (or quasi-ribbon) elements is determined by square roots of $g$.

Proposition 6. Suppose that $(H, R)$ is a co-Frobenius quasitriangular Hopf algebra with antipode $S$ over a field $k$, Suppose further that $H$ is unimodular and let $g$ be the distinguished group-like element of $H$. Then:

1) $(H, R)$ has a quasi-ribbon element if and only if $l^{2}=g$ for some $l \in G(H)$;

2) $(H, R)$ has a ribbon element if and only if $l^{2}=g$ for some $l \in G(H)$ which satisfies $S^{2}(a)=l a l^{-1}$ for all $a \in H$.

Proposition 7. Suppose that $(H, R)$ is a co-Frobenius quasitriangular Hopf algebra with antipode $S$ over a field $k$, suppose further that $H$ and $H^{*}$ are both unimodular Then:

1) $u$ is a quasi-ribbon element of $(H, R)$;

2) $u$ is a ribbon element of $(H, R)$ if and only if $S^{2}=I$.

\section{Acknowledgements}

The author is supported by the NSFC project 10826037.

\section{References}

[1] B. I. P. Lin, "Semiperfect Coalgebras," Journal of Algebra, Vol. 49, No. 2, 1977, pp. 357-373. 
[2] Y. Doi, "Homological Coalgebra," Journal of the Mathematical Society of Japan, Vol. 33, No. 1, 1981, pp. 3150.

[3] A. van Daele, "An Algebraic Framework for Group Duality," Advances in Mathematics, Vol. 140, No. 2, 1998, pp. 323-366.

[4] N. Y. Reshetikhin and V. G. Turaev, "Ribbon Graphs and their Invariants Derived from Quantum Groups," Communications in Mathematical Physics, Vol. 127, No. 1, 1990, pp. 1-26.

[5] N. Y. Reshetikhin and V. G. Turaev, "Invariants of 3-Manifolds via Link Polynomials and Quantum Groups," Invented Mathematics, Vol. 103, No. 3, 1991, pp. 547-597.

[6] R. Kirby and P. Melvin, "The 3-Manifolds Invariants of Witten and Reshetikhin-Turaev for sl (2, C)," Invented Mathematics, Vol. 105, No. 3, 1991, pp. 473-545.

[7] M. Beattie, D. Bulacu and B. Torrecillas, "Radford's s4 Formula for Co-Frobenius Hopf Algebras," Journal of Algebra, Vol. 307, No. 1, 2007, pp. 330-342.

[8] M. Beattie, S. Dascalescu, L. Grunenfelder and C. Nastasescu, "Finiteness Conditions, Co-Frobenius Hopf Algebras and Quantum Groups," Journal of Algebra, Vol.
200, No. 1, 1998, pp. 312- 333.

[9] M. Beattie, S. Dascalescu and S. Raianu, "Galois Extensions for Co-Frobenius Hopf Algebras," Journal of Algebra, Vol. 198, No. 1, 1997, pp. 164-183.

[10] L. H. Kauffman and D. E. Radford, "A Necessary and Sufficient Condition for a Finite-Dim-Ensional Drinfeld Double to be a Ribbon Hopf Algebras," Journal of Algebra, Vol. 159, No. 1, 1993, pp. 98-114.

[11] V. G. Drinfeld, "On Almost Cocommutative Hopf Algebras," Leningrad Mathematical Journal, Vol. 1, No. 2, 1990, pp. 321-342.

[12] D. E. Radford, "On the Antipode of a Quasitriangular Hopf Algebras," Journal of Algebra, Vol. 151, No. 1, 1992, pp. 1-11.

[13] M. Beattie and D. Bulacu, "On the Antipode of a Co-Frobenius (Co) Quasitriangular Hopf Algebras," Communications in Algebra, Vol. 37, No. 9, 2009, pp. 2981-2993.

[14] N. Y. Reshetikhin and V. G. Turaev, "Invariants of 3-Manifolds via Link Polymonials and Quantum Groups," Communications in Mathematical Physics, Vol. 127, No. 1, 1990, pp. 7-26. 